\title{
Itraconazole Coated Super Paramagnetic Iron Oxide Nanoparticles for Antimicrobial Studies
}

\author{
Antony V Samrot ${ }^{1 * \mathbb{D}}$, Chamarthy Sai Sahithya ${ }^{2 \mathbb{D}}$, Durga Sruthi $\mathbf{P}^{2}$, Jenifer Selvarani ${ }^{2 \mathbb{D}}$, Raji $\mathbf{P}^{2}$, \\ Prakash $P^{2}$, Paulraj Ponnaiah ${ }^{1}$, Iyappan Petchi ${ }^{1}$, Sajeesh Pattammadath ${ }^{1}{ }^{(\mathbb{D}}$, Sajna Keeyari \\ Purayil ${ }^{1}$ (D), Pazhayakath Thevarkattil Mohamed Javad ${ }^{1}$, Thirumurugan $\mathbf{R}^{3}$, Kasirajan Kasipandian ${ }^{4}$ \\ 1 Department of Biomedical Sciences, Faculty of Medicine and Biomedical Sciences, MAHSA University, Jenjarom, \\ Selangor - 42610, Malaysia \\ 2 Department of Biotechnology, Sathyabama Institute of Science and Technology, Jeppiar Nagar, Chennai - 600 119, Tamil \\ Nadu, India \\ 3 Department of Transfusion Medicine, JIPMER, Puducherry - 605 006, India \\ 4 Department of Information Technology, MAHSA University, Jenjarom, Selangor, Malaysia \\ * Correspondence: antonysamrot@gmail.com;
}

Scopus Author ID 36100751800

Received: 25.04.2020; Revised: 8.05.2020; Accepted: 8.05.2020; Published: 12.05.2020

\begin{abstract}
In this present study, Superparamagnetic Iron Oxide Nanoparticles (SPIONs) were produced using $\mathrm{FeCl}_{3}$ and $\mathrm{FeCl}_{2}$ which were reduced to iron oxides using $\mathrm{NaOH}$ and ammonia solution (chemical co-precipitation). These naked SPIONs were further fabricated to form drug laden core-shell for controlled drug release and delivery. The fabrication was achieved by subjugating the naked SPIONs for oleic acid functionalization, drug tagging (Itraconazole) and finally encapsulated with a microbial derived polyester namely Polyhydroxybutyrate (PHB). Every stage of fabrication was characterized by scanning electron microscopy (SEM). The core-shell produced was checked for drug release kinetics, antibacterial and antifungal activities. These synthesized core-shells were carrying the drug and showed a slow drug release profile. The antimicrobial studies against bacteria - Pseudomonas aeruginosa \& Brevibacillus brevis and fungi - Candida albicans by diffusion method proved that the core-shells inhibited bacterial and fungal activity. Furthermore, the naked SPIONs was found to be a good contrasting agent in X-ray imaging.
\end{abstract}

Keywords: SPIONs; Core-shell formation; antimicrobial activity.

(C) 2020 by the authors. This article is an open access article distributed under the terms and conditions of the Creative Commons Attribution (CC BY) license (https://creativecommons.org/licenses/by/4.0/).

\section{Introduction}

During these last decades, the growth of nanotechnology is humungous. Various types of nanoparticles are there, which are differentiated from each other by their nature and properties. Amongst all, metallic nanoparticles have tremendous scope and application in various fields including medicine, pharmaceutics, environmental remediation and others. Physical, chemical and biological properties of these nanoparticles aid them to be applied in various applications including theranostic, drug delivery, biosensing, imaging etc [1]. Metallic nanoparticles including gold, silver, Zn, iron oxides etc are most often used nanoparticles and proven to have various activities including anticancer activity, antibacterial, environmental bioremediation etc[2-10]. Amongst all these, iron oxides are cheaper to produce and versatile in action, moreover, they are biocompatible too. Iron oxides with magnetic properties are commonly called SPIONs (SuperParamagnetic Iron oxide nanoparticles), these nanoparticles are mostly exploited in imaging, drug delivery and so on. The major issue is its unstable in 
nature, which can be overruled by functionalizing them with surfactant like SDS [11] or any other molecule like oleic acids [12]. Functionalization is usually performed to increase the colloidal stability as well as to prevent aggregation. The preparation of SPIONs is commonly done by chemical co-precipitation which is economically and sometimes they are also biologically derived [3]. On surface modification, the surface of these SPIONs becomes more functional to interact with further coating molecules. In this way, SPIONs are customized to form as effective drug nanocarriers with its characteristic feature of being able to be controlled by the applied magnetic field. In our earlier publications, we had prepared different types of SPIONs with varied precursors and reducing agents. The potential of each SPIONs was studied for different applications such as drug carriers [13], imaging [3,14], environmental bioremediation $[6,15]$.

Here, it is an initial effort to produce SPIONs core-shells for drug tagging and drug delivery. Encapsulation with a biological source will help in increasing the biocompatibility and to confine the tagged drug onto the core-shells. Therefore, commercially available Polyhydroxybutyrate (PHB) was employed for encapsulating the core-shells. PHB is one of the most common polyhydroxyalkanoate (PHA) molecule produced by bacteria under stress conditions [16]. It is widely used as a natural biodegradable polymer for delivery drug / pesticide, tissue engineering and in medical devices [17 -19]. In this study, SPIONs were synthesized by co-precipitation method and coated with Itraconazole followed by PHB encapsulation. The drug release kinetics and efficiency of these core-shells were checked by antimicrobial studies. Also, chicken eggs were treated with the chemically prepared naked SPIONs to assess its competence as a contrasting agent in X-ray imaging.

\section{Materials and Methods}

\subsection{Materials}

All the solvents were all analytical grade. The following chemicals were purchased with AR grade - Ferrous chloride (LOBACHEMIE), Ferric chloride (LOBACHEMIE), Ammonium solution (SRL), Sodium hydroxide (SRL), Hydrochloric Acid. PHB was obtained from Sigma Chemicals. The whole study was done using nitrogen purged MilliQ water.

\subsection{Methods.}

\subsubsection{Synthesis of SPIONs.}

Synthesis of SPIONs was done using the protocol followed by Samrot et al [15]. The precursor solution of $1 \mathrm{M}$ of $\mathrm{FeCl}_{3}$ and $3 \mathrm{M}$ of $\mathrm{FeCl}_{2}$ were prepared in nitrogen purged Millipore water. Equal volumes $(10 \mathrm{ml})$ of the two precursor solutions were mixed together and heated to $60{ }^{\circ} \mathrm{C}$. To this, $15 \mathrm{ml}$ of $8 \mathrm{M} \mathrm{NaOH}$ and $5 \mathrm{ml}$ of Ammonia solution were added in drops simultaneously and stirred vigorously. The temperature was maintained throughout the reaction. The black precipitate formed was settled down by applying a magnetic field. Thus separated black precipitate was washed with Nitrogen purged MilliQ water for multiple times to bring to neutral $\mathrm{pH}$ and later lyophilized to form dry powders.

\subsubsection{Fabrication and characterization of drug loaded core-shells SPIONs.}

Oleic acid functionalization, tagged with itraconazole and encapsulation with PHB was done in accordance with Sruthi et al [12]. Oleic acid solution in the ratio of 1:1 in ethanol was 
prepared for $20 \mathrm{ml}$ and $20 \mathrm{mg}$ of SPIONs were added. The prepared mixture was placed in rotary shaker for $5 \mathrm{~h}$ and then sonicated for $30 \mathrm{mins}$. Thus functionalized SPIONs were utilized for coating with Itraconazole. The drug solution was prepared at 1:2 ratio in ethanol for $30 \mathrm{ml}$ and added with $20 \mathrm{mg}$ of functionalized SPIONs. The prepared solution was left to interact with continuously stirring for $18 \mathrm{~h}$. Further encapsulation of drug tagged SPIONs with PHB was successfully achieved by following Sruthi et al [12]. The powdered sample was characterized at every stage using Scanning Electron Microscopy (SEM).

\subsubsection{Drug Release Kinetics.}

After encapsulation with the biopolymer, the rate of drug release was determined by dialysis method [20-23], $10 \mathrm{mg}$ of drug loaded SPIONs were dialysed against $50 \mathrm{ml}$ PBS buffer [23]. At every time interval, $1 \mathrm{ml}$ of sample dipped buffer was collected and OD value read at $267 \mathrm{~nm}$ wavelength ( $\lambda$ max of Itraconazole).

\subsubsection{Antimicrobial Activity.}

Agar well diffusion method was used to determine the anti-microbial activity against bacteria [24, 25] - Pseudomonas aeruginosa \& Brevibacillus brevis and fungi - Candida albicans. The PHB encapsulated SPIONs were used in different concentrations such as $5 \mu \mathrm{g}$, $10 \mu \mathrm{g}, 15 \mu \mathrm{g}$ and $20 \mu \mathrm{g}$ against a positive and negative controls. The plates were incubated at $37{ }^{0} \mathrm{C}$ for $24 \mathrm{~h}$.

\subsubsection{X-Ray imaging.}

The X-ray imaging ability of SPIONs was tested on chicken's eggs to study its application as a contrasting agent. The experiment was carried out as described by Justin et al [14]. With the help of a syringe, $2 \mathrm{ml}$ of the glaire material was removed from the egg. It was replaced with $2 \mathrm{ml}$ of naked SPIONs solution $(100 \mathrm{mg} / \mathrm{ml})$ and gently shaken to get the particles dispersed inside the egg thoroughly. After it is done, the egg was exposed to X-ray imaging

\section{Results and Discussion}

\subsection{Scanning Electron Microscopy (SEM).}

SEM results show that the SPIONs, Functionalized SPIONs, Drug coated SPIONs and PHB encapsulated SPIONs have a size range of 40 - $45 \mathrm{~nm}$ (Fig.1a), 40 - 65nm (Figure 1b), 50-70 nm (Figure 1c) and $100-130 \mathrm{~nm}$ (Figure 1d) respectively. As the result of coating, the nanoparticles seemed to increase in size gradually. Ultimately the PHB encapsulated core-shell reached a size range between 100 and 130nm. They were also seen to have spherical shape.

\subsection{Drug Release Kinetics.}

From the drug release kinetics study, it was clear that the drug is released in a sustainable manner overall, even with the presence of few troughs (Figure 2). It was seen to maintain a steady increase after $75^{\text {th }}$ min up until $165^{\text {th }}$ min. PHB nanoparticle is having the character of releasing drug slowly and steadily [26]. 


\subsection{Anti-microbial study.}

Anti-microbial study performed using agar well diffusion method showed that there was no activity seen against the bacteria species, Pseudomonas aeruginosa \& Brevibacillus brevis. There was a significant zone of inhibition for Core-shell SPIONs at concentrations 15 \& $20 \mathrm{mg}$ i.e., 1.7 and $2 \mathrm{~cm}$ diameter (Table 1). A similar result was found by Sruthi et al [12].
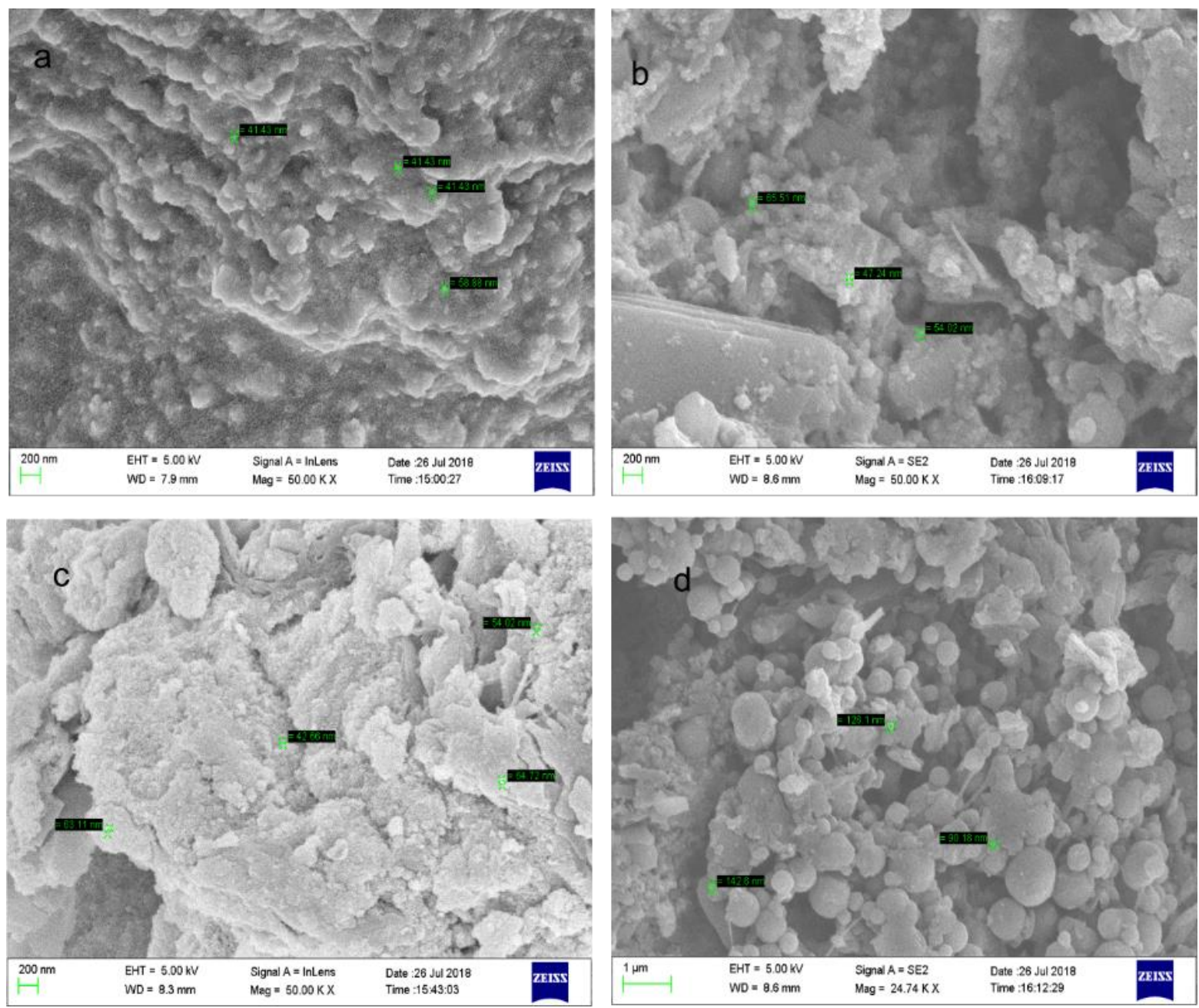

Figure 1. a) SPIONs b) Functionalized with oleic acids c) coated with itraconazole d) encapsulated with PHB.

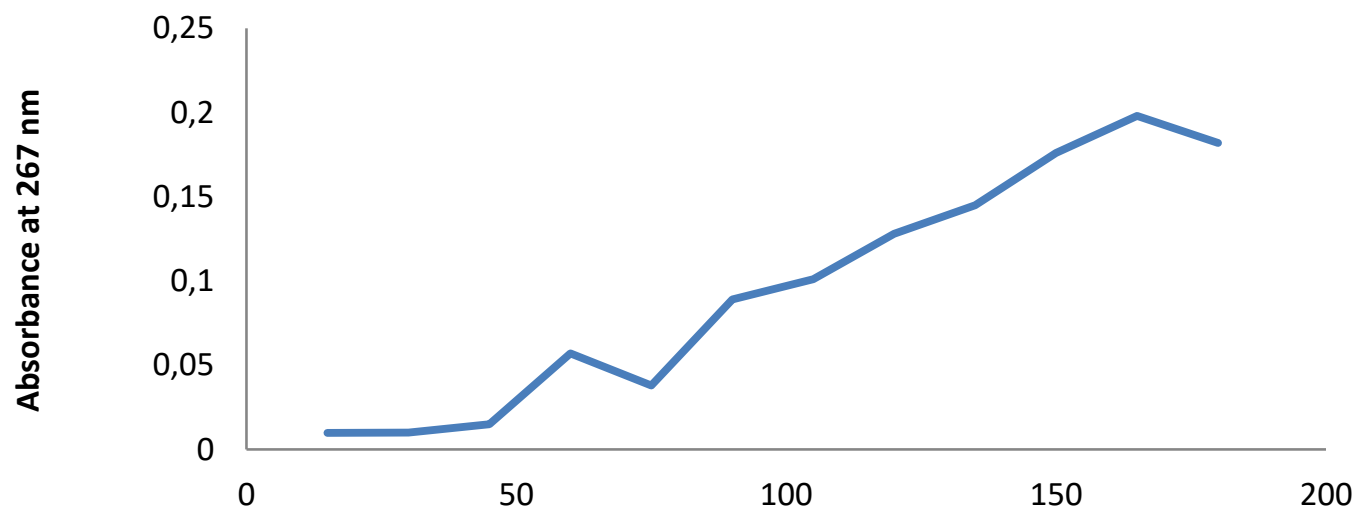

Time (min)

Figure 2. Drug release pattern of PHB encapsulated core-shell SPIONs.

c) Candida albicans. 
Table 1. Antimicrobial activity of drug encapsulated Core shell SPIONs.

\begin{tabular}{c|c|c|c}
\hline \multirow{2}{*}{ Concentration $(\mu \mathrm{g} / \mathrm{ml})$} & \multicolumn{3}{|c}{ ZONE OF INHIBITION $(\mathrm{cm})$} \\
\cline { 2 - 4 } & $\begin{array}{c}\text { Pseudomonas } \\
\text { aeruginosa }\end{array}$ & Brevibacillus brevis & Candida albicans \\
\hline POSITIVE CONTROL & 4 & 3.5 & 2.8 \\
\hline NEGATIVE CONTROL & - & - & - \\
\hline 5 & - & - & - \\
\hline 10 & - & - & 1.7 \\
\hline 15 & - & - & 2 \\
\hline 20 & 3.2 & 0.3 & \\
\hline
\end{tabular}
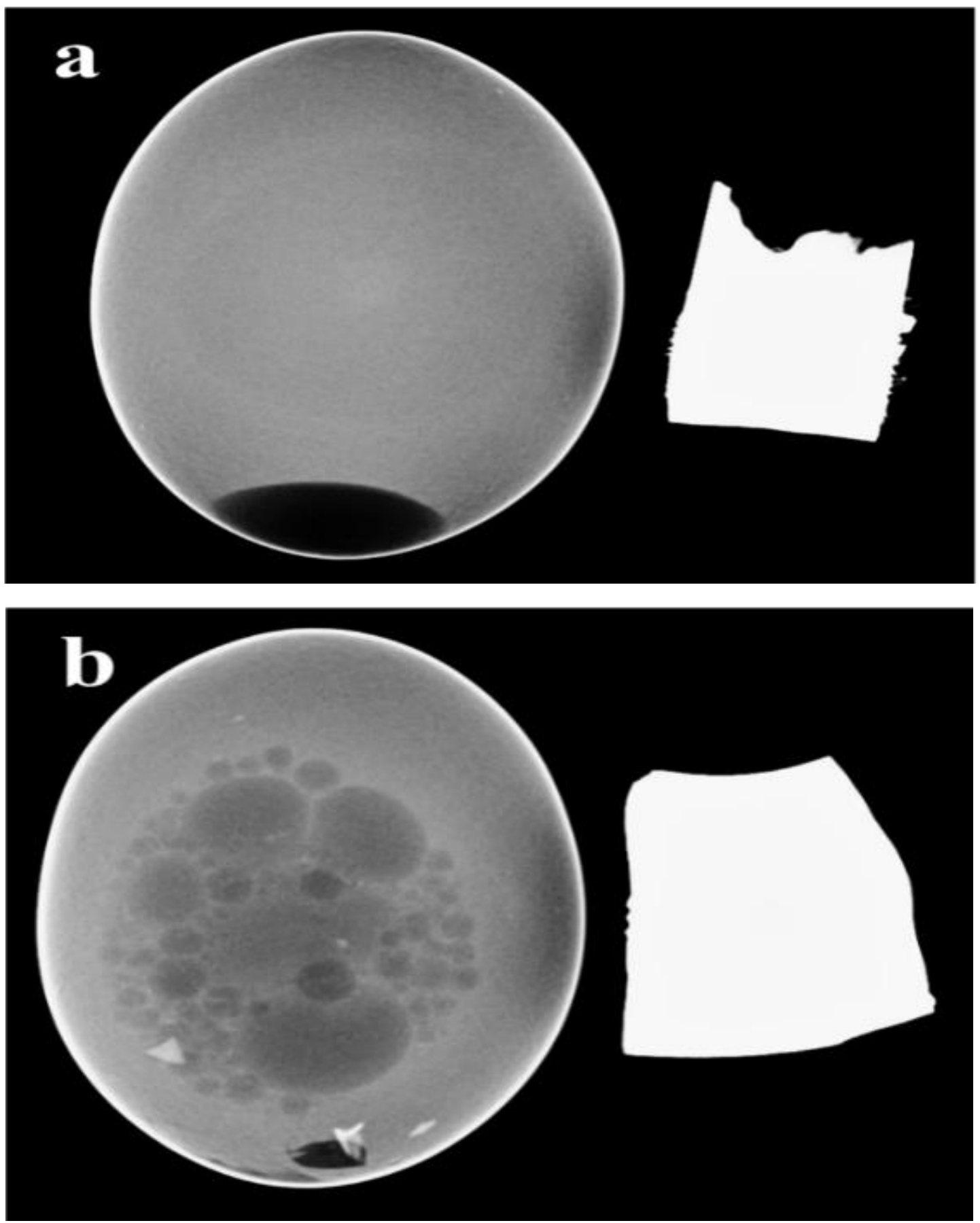

Figure 3. Imaging of egg under X-ray a) Control egg without SPIONs b) Egg injected with SPIONs. 


\subsection{X-ray Imaging.}

Internal components of the egg could be visualized more clearly when injected with SPIONs (Figure 3). Hence SPIONs has the tendency to enhance the x-ray imaging. In our earlier report with quail's egg, SPIONs treated egg showed similar contrasting effect with increased quality of imaging $[12,14,27]$. And with the help of an externally applied magnetic field, it is even possible to retrieve the SPIONs from the treated egg. There are more reports where these iron oxides were used in various pharmaceutical industries [28].

\section{Conclusions}

The synthesized core-shell SPIONs were successfully loaded with the drug, itraconazole and further encapsulated with PHB. The drug-encapsulated SPIONs reached to a size of 100-130nm. These drug laden core-shells expressed steady drug kinetics and released out the maximum drug at $160^{\text {th }} \mathrm{min}$. The core-shell SPIONs exhibited antibacterial activity only against P.aeruginosa and antifungal activity against C.albicans at the highest concentration of $20 \mu \mathrm{g} / \mathrm{ml}$. The chemically synthesized naked SPIONs were found to improve the X-ray visualization by acting as a good contrasting agent.

\section{Funding}

This research received no external funding.

\section{Acknowledgments}

This research has no acknwoledgments.

\section{Conflicts of Interest}

The authors declare no conflict of interest.

\section{References}

1. Shnoudeh, A.J.; Hamad, I.; Abdo, R.W.; Qadumii, L.; Jaber, A.Y.; Surchi, H.S.; Alkelany, S.Z. Synthesis, Characterization, and Applications of Metal Nanoparticles. Biomaterials and Bionanotechnology Advances in Pharmaceutical Product Development and Research 2019, 527-612, https://doi.org/10.1016/B978-0-12814427-5.00015-9.

2. Raji, P.; Samrot, A.V.; Bhavya, K.S.; Sharan, M.; Priya, S.; Paulraj, P. Greener approach for leather tanning using less chrome with plant tannins and tannins mediated nanoparticles. J Clust Sci. 2019, https://doi.org/10.1007/s10876-019-01597-6.

3. Samrot, A.V.; SenthilKumar, P.; Rashmitha, S.; Veera, P.; Sahithya, C.S. Azadirachta indica influenced biosynthesis of superparamagnetic iron oxide nanoparticles and their applications in tannery water treatment and x-ray imaging. Nanostructure in Chemistry 2018, 8, 343-351, https://doi.org/10.1007/s40097-0180279-0.

4. Samrot, A.V.; Shobana, N.; Kumar, S.S.; Narendrakumar, G. Production, optimization and characterisation of chitosanase of Bacillus sp and its applications in nanotechnology. J Clust Sci. 2019, 30, 607-620, https://doi.org/10.1007/s10876-019-01520-z.

5. Raji, P.; Samrot, A.V.; Keerthana, D.; Karishma, S. Antibacterial Activity of Alkaloids, Flavonoids, Saponins and Tannins Mediated Green Synthesised Silver Nanoparticles Against Pseudomonas aeruginosa and Bacillus subtilis. J Clust Sci. 2019, 30, 881-895, https://doi.org/10.1007/s10876-019-01547-2.

6. Samrot, A.V.; Shobana, N.; Sruthi, D.P.; Sahithya, C.S. Utilization of chitosan coated superparamagnetic iron oxide nanoparticles for chromium removal. Appl. Water Sci. 2018, 8, 192, https://doi.org/10.1007/s13201-018-0841-4. 
7. Samrot, A.V.; Raji, P.; Selvarani, A.J.; Nishanthini, P. Antibacterial activity of some edible fruits and its green synthesized silver nanoparticles against uropathogen - Pseudomonas aeruginosa SU 18. Biocatalysis and Agricultural Biotechnology 2018, 16, 253-270, https://doi.org/10.1016/j.bcab.2018.08.014

8. Samrot, A.V.; Silky; Ignatious, C.V.; Raji, P.; SaiPriya, C.; Selvarani, A.J. Bioactivity Studies of Datura metel, Aegle marmelos, Annona reticulata and Saraca indica and their Green Synthesized Silver Nanoparticle. J Pure Appl Microbiol. 2019, 13, https://doi.org/10.22207/JPAM.13.1.

9. Gandham, R.G.; Raji, P.; Rohan, B.D.; Kumar, D.M.; Sharma, K.V.; Keerthana, D.; Karishma, S.; Iyappan, P.; Thirumurugan, R.; Samrot, A.V.; Ponniah, P.; Pattammadath, S.; Purayil, K.P.; Javad, P.T.M.; Prakash, P. Green Synthesis and antibacterial activity studies of silver nanoparticles from the aqueous extracts of Cassia alata. Letters in Applied NanoBioScience 2020, 9, 1037 - $1041 . \quad$ DOI: 10.33263/LIANBS92.10371041

10. Kumar, D.M.; Raji, P.; Rohan, B.D.; Gandham, R.G.; Sharma, K.V.; Keerthana, D.; Karishma, S.; Samrot, A.V.; Ponniah, P.; Thirumurugan, R.; Pattammadath, S.; Selvarani, A.J.; Prakash, P. Green Synthesis and antibacterial activity studies of silver nanoparticles from the aqueous extracts of Thespesia populnea. Letters in Applied NanoBioScience 2020, 9, 931 - 934. https://doi.org/10.33263/LIANBS91.931934.

11. Justin, C.; Samrot, A.V.; Sruthi, P.D.; Sahithya, C.S.; Bhavya, K.S.; Saipriya, C. Preparation, characterization and utilization of core-shell super paramagnetic iron oxide nanoparticles for curcumin delivery. PLoS ONE 2018, 13, https://doi.org/10.1371/journal.pone.0200440.

12. Sruthi, P.D.; Sahithya, C.S.; Justin, C.; SaiPriya, C.; Bhavya, K.S.; Senthilkumar, P.; Samrot, A.V. Utilization of Chemically Synthesized Super Paramagnetic Iron Oxide Nanoparticles in Drug Delivery, Imaging and Heavy Metal Removal. J Clust Sci. 2018, 30, 11-24, https://doi.org/10.1007/s10876-018-14547.

13. Justin, C.; Samrot, A.V.; Annamalai, A.A.; Bhattacharya, R.; Sathiyamoorthy, P; Sahithya, C.S. Biopolymer Coated Core-shell Magnetite Nanoparticles for Rifampicin Delivery. Orient. J. Chem. 2018, 34, 2389-2396, https://doi.org/10.13005/ojc/340521.

14. Justin, C.; Philip, S.A.; Samrot, A.V. Synthesis and characterization of superparamagnetic iron-oxide nanoparticles (SPIONs) and utilization of SPIONs in X-ray imaging. Appl Nanosci. 2017, 7, 463-475, https://doi.org/10.1007/s13204-017-0583-X.

15. Samrot, A.V.; Sahithya, C.S.; Selvarani, A.J.; Pachiyappan, S.; Kumar, S.S. Surface-Engineered SuperParamagnetic Iron Oxide Nanoparticles for Chromium Removal. Int J Nanomedicine 2019, 8105-8119, https://doi.org/10.2147/IJN.S214236.

16. Paulraj, P.; Shukri, H.; Chandramohan, M.; Narendrakumar, G.; Samrot, A.V. Utilization of palm oil mill effluent and clindamycin for optimization of polyhydroxy [r] alkanoates production. Biointerface Research in Applied Chemistry 2020, 10, 4740 - 4746. DOI: 10.33263/BRIAC101.740746

17. Shrivastav, A.; Kim, H.Y.; Kim, Y.R. Advances in the Applications of Polyhydroxyalkanoate Nanoparticles for Novel Drug Delivery System. Biomed Research International 2013, 2013, https://doi.org/10.1155/2013/581684.

18. Samrot, A.V.; Angalene, J.L.A.; Roshini, S.M.; Stefi, S.M.; Preethi, R.; Raji, P.; Kumar, M.A.; Kumar, S.S. Purification, characterization and exploitation of Azadirachta indica gum for the production of drug loaded nanocarrier. Mater. Res. Express 2020, 7, 055007.

19. Samrot, A.V.; Bhavya, K.S.; Angalene, J.L.A.; Roshini, S.M.; Preethi, R.; Steffi, S.M.; Raji, P.; Kumar, S.S. Utilization of Gum Polysaccharide of Araucaria Heterophylla and Azadirachta Indica for Encapsulation of Cyfluthrin Loaded Super Paramagnetic Iron Oxide Nanoparticles for Mosquito Larvicidal Activity. Int J Biol Macromol. 2020, 19, doi: 10.1016/j.ijbiomac.2019.10.232. DOI: 10.1088/2053-1591/ab8b16

20. Samrot, A.V.; Angalene, J.L.A.; Roshini, S.M.; Stefi, S.M.; Preethi, R.; Raji, P.; Madankumar, A.; Paulraj, P.; Kumar, S.S. Purification, characterization and utilization of polysaccharide of Araucaria heterophylla gum for the synthesis of curcumin loaded nanocarrier. Int J Biol Macromol. 2019, 140, 393-400. DOI: 10.1016/j.ijbiomac.2019.08.121

21. Samrot, A.V.; Akanksha, Jahnavi, T.; Padmanaban, S.; Philip, S.A.; Burman, U.; Rabel, A.M. Chelators influenced synthesis of chitosan-carboxymethyl cellulose microparticles for controlled drug delivery. Appl Nanosci. 2016, 6, 1219-1231, https://doi.org/10.1007/s13204-016-0536-9.

22. Samrot, A.V.; Burman, U.; Philip, S.A.; Shobana, N.; Chandrasekaran, K. Synthesis of curcumin loaded polymeric nanoparticles from crab shell derived chitosan for drug delivery. Informatics in Medicine Unlocked 2018, 10, 159-182, https://doi.org/10.1016/j.imu.2017.12.010.

23. Senthilkumar, P.; Dawn, S.S.; Saipriya, C.; Samrot, A.V. Surfactant (span20) influenced synthesis of polyhydroxybutyrate nanoparticles for hydrophobic drug delivery. Rasayan Journal of Chemistry 2018, 11, $1686-1695$

24. Parveen, S.; Wania, A.H.; Shahb, M.A.; Devib, H.S.; Bhata, M.Y.; Kokaa, J.A. Preparation, characterization and antifungal activity of iron oxide nanoparticles. Microb. Pathog. 2018, 115, 287-292, https://doi.org/10.1016/j.micpath.2017.12.068.

25. Hussein-Al-Ali, S.H.; El-Zowalaty, M.E.; Hussein, M.Z.; Geilich, B.M.; Webster, T.J. Synthesis, characterization, and antimicrobial activity of an ampicillin-conjugated magnetic nano antibiotic for medical applications. Int J Nanomedicine 2014, 9, 3801-3814, https://doi.org/10.2147/IJN.S61143. 
26. Senthilkumar, P.; Dawn, S.S.; Samanvitha, S.K.; Saipriya, C.; Samrot, A.V. Surfactant Mediated Synthesis of Polyhydroxybutyrate (PHB) Nanoparticles for Sustained Drug Delivery. IET nanobiotechnology 2019, https://doi.org/10.1049/iet-nbt.2018.5053.

27. Samrot, A.V.; Bhavya, K.S.; Sruthi, D.P.; Raji, P.; Paulraj, P. Synthesis of SPIONs to deliver drug in-vitro and to use as contrasting agent. International Journal of Advanced Research in Engineering and Technology (IJARET) 2020, 11, 200-208,

28. Mohamad, A.; Rizwan, M.; Keasberry, N.A., Ahmed, M.N. Fabrication of label-free electrochemical food biosensor for the sensitive detection of ovalbumin on nanocomposite-modified graphene electrode. Biointerface Research in Applied Chemistry 2019, 9, 4655 - 4662. http://doi.org/10.33263/BRIAC96.655662 